\begin{tabular}{|l|c|c|c|l|l|}
\hline \multirow{2}{*}{$\sim$} & A P H W & \multicolumn{1}{|c|}{12080} & \multirow{2}{*}{ WILEY } & Dispatch: 1.10.16 & CE: Wiley \\
\cline { 2 - 3 } & Journal Code & Manuscript No. of pages: 19 & PE: Sathya Priya M. \\
\hline
\end{tabular}

APPLIED PSYCHOLOGY: HEALTH AND WELL-BEING, 2016

doi:10.1111/aphw.12080

\title{
Too Depleted to Try? Testing the Process Model of Ego Depletion in the Context of Unhealthy Snack Consumption
}

\author{
Ashleigh Haynes* and Eva Kemps \\ School of Psychology, Flinders University, Adelaide, Australia
}

\author{
Robyn Moffitt \\ School of Applied Psychology, Griffith University, Queensland, Australia \\ Menzies Health Institute, Queensland, Australia
}

Background: The process model proposes that the ego depletion effect is due to (a) an increase in motivation toward indulgence, and (b) a decrease in motivation to control behaviour following an initial act of self-control. In contrast, the reflective-impulsive model predicts that ego depletion results in behaviour that is more consistent with desires, and less consistent with motivations, rather than influencing the strength of desires and motivations. The current study sought to test these alternative accounts of the relationships between ego depletion, motivation, desire, and self-control. Methods: One hundred and fifty-six undergraduate women were randomised to complete a depleting e-crossing task or a non-depleting task, followed by a lab-based measure of snack intake, and self-report measures of motivation and desire strength. Results and Conclusions: In partial support of the process model, ego depletion was related to higher intake, but only indirectly via the influence of lowered motivation. Motivation was more strongly predictive of intake for those in the non-depletion condition, providing partial support for the reflective-impulsive model. Ego depletion did not affect desire, nor did depletion moderate the effect of desire on intake, indicating that desire may be an appropriate target for reducing unhealthy behaviour across situations where self-control resources vary.

Keywords: desire, ego depletion, food intake, motivation, self-control

\section{INTRODUCTION}

Exertion of self-control over eating behaviour is required on a daily basis, due to the availability and affordability of immediately rewarding, but unhealthy, snack

* Address for correspondence: Ashleigh Haynes, Psychological Sciences, University of Liverpool, Eleanor Rathbone Building, Liverpool L69 7ZA, UK. Email: a.haynes@liverpool.ac.uk 
foods. These foods present a choice between satisfying urges to consume the food for immediate hedonic gratification, and controlling those urges to act in a manner consistent with longer-term goals, like healthy weight management. Following one act of self-control, the ability to enact self-control is temporarily depleted, such that self-control attempts are less likely to be successful, an effect termed "ego depletion" (Baumeister, Bratslavsky, Muraven, \& Tice, 1998). However, the size, replicability, and the conditions under which the ego depletion effect can be observed are subjects of ongoing enquiry (Carter \& McCullough, 2014; Hagger et al., 2015; Job, Dweck, \& Walton, 2010). Understanding more about the mechanisms underlying ego depletion (including the variables that interact with and mediate the effect) may contribute to an understanding of how to prevent self-control failures and improve outcomes in a variety of behavioural domains, including academic and work performance, overspending, smoking, alcohol and drug abuse, relationship problems, as well as unhealthy snacking.

\section{The Reflective-Impulsive Model and Ego Depletion}

According to traditional accounts, self-control involves the effortful inhibition of impulses toward behaviour that is immediately rewarding (e.g. consuming unhealthy food) to enact behaviour consistent with motivations toward longterm goals (e.g. weight management) (Fujita, 2011; Muraven \& Baumeister, 2000). Ego depletion is thought to alter the relative influence of impulses and goal motivations on behaviour, such that when depleted, behaviour is driven to a greater extent by immediate desires and impulses, and to a lesser extent by motivations toward long-term goals (Hofmann, Friese, \& Wiers, 2008; Strack \& Deutsch, 2004). For example, the reflective-impulsive model posits that ego depletion affects the relative influence of two opposing systems on behaviour, the impulsive and reflective systems. On the one hand, impulsive influences operate relatively automatically to drive behaviour toward hedonically rewarding stimuli, and can include a conscious desire to indulge in unhealthy food (Milyavskaya, Inzlicht, Hope, \& Koestner, 2015; Strack \& Deutsch, 2004). On the other hand, the reflective system operates in a more reasoned manner, guiding behaviour in line with motivations and long-term goals. However, the reflective system requires sufficient self-control resources to influence behaviour (Deutsch \& Strack, 2006; Hofmann et al., 2008; Strack $\&$ Deutsch, 2004). In line with this account, ego depletion interacts with both desire for immediately gratifying unhealthy snack food (an impulsive influence) and motivation toward a long-term goal (a reflective influence) to predict self-control of eating behaviour. Specifically, when depleted, behaviour is more strongly predicted by desire, and when not depleted, behaviour is more strongly predicted by motivations. 


\section{The Process Model of Ego Depletion}

The process model proposed by Inzlicht and Schmeichel (2012) presents an alternative account of how ego depletion operates in relation to motivation and desire in predicting self-control outcomes. According to this model, the detriment to self-control performance following an initial self-control act is due to a shift in priorities and motivation. After one act of self-control, individuals become more motivated to indulge and satisfy immediate desires, and simultaneously become less motivated to control their urges. Inzlicht and Schmeichel argue that it is adaptive for individuals to balance periods of control and leisure, so that after enacting control, they become more motivated toward rest and indulgence of impulses. Thus, according to the process model, ego depletion does not moderate the effect of desire and motivation toward longer-term goals on behaviour (as predicted by traditional accounts of self-control); instead, the model proposes that ego depletion results in a stronger motivation to seek immediate gratification, and a reduced motivation to control those urges.

\section{Relationship between Ego Depletion and Desire for Unhealthy Stimuli}

Although Inzlicht, Schmeichel, and Macrae (2014) suggest that depletion increases desire for unhealthy or appetitive stimuli, the evidence in support of an increase in desire or motivation toward indulgence accompanying depletion is mixed. For example, Schmeichel, Harmon-Jones, and Harmon-Jones (2010) showed that depletion increased incentive sensitivity (i.e. increased motivation toward, pursuit of, and positive feeling in response to obtaining incentives) and facilitated the perception of reward-related stimuli (i.e. participants were quicker to detect a dollar sign in an array of symbols when depleted). In addition, Wagner, Altman, Boswell, Kelley, and Heatherton (2013) found that dieters who completed a depletion task showed greater activity in the orbitofrontal cortex (an area of the brain thought to be associated with processing reward value) when viewing pictures of food, than did dieters who were not depleted.

By contrast, other studies have found that rather than depletion resulting in stronger desire, desire is more influential on behaviour when individuals are depleted. Although not quite the same as motivation toward approaching or indulging in unhealthy foods, positive implicit attitudes toward such foods have been shown to predict stronger desire and preference for those foods (e.g. Dube, 2007; Friese, Hofmann, \& Wanke, 2008; Haynes, Kemps, Moffitt, \& Mohr, 2014; Perugini, 2005). Hofmann, Rauch, and Gawronski (2007) found that for participants in a depletion condition, more positive implicit food evaluations predicted higher intake of candy in a lab-based taste-test, whereas for those in a non-depletion condition, implicit food attitudes did not predict intake. These results therefore suggest that when depleted, individuals lack the ability to 
overcome impulses or desires toward unhealthy behaviour. Friese et al. (2008) reported similar findings: positive implicit evaluations of potato chips predicted higher consumption in a taste-test, but only when participants were depleted. Furthermore, Hofmann, Vohs, and Baumeister (2012) conducted an experience sampling study requiring participants to respond to smartphone-administered questionnaires multiple times a day over a one-week period. Participants were asked to report whether they had recently experienced a desire from a variety of domains (e.g. food, spending, etc.), whether they had attempted to resist that desire, and whether or not they had enacted behaviour consistent with their desire. Similar to the findings of studies examining implicit food attitudes, depletion (inferred from a recent attempt at self-control of desire) predicted lower success in overcoming desire. These findings are consistent with traditional accounts of self-control such as the reflective-impulsive model, suggesting that ego depletion allows desire to more strongly influence behaviour, rather than depletion increasing the strength of desire.

\section{Relationship between Ego Depletion and Motivation to Control Behaviour}

The reflective-impulsive and process models also make competing predictions about how motivations to control behaviour are involved in ego depletion effects. According to the reflective-impulsive model, ego depletion results in behaviour that is more strongly driven by desires for immediate indulgence, and to a lesser extent by motivations toward long-term goals. By contrast, the process model not only proposes that ego depletion shifts motivation toward indulgence (thus increasing desire), but also that it reduces motivation to control behaviour in line with long-term goals. In support of the reflective-impulsive model, the results of an experience sampling study by Hofmann et al. (2012) showed that depletion did not increase the likelihood of attempting to resist a desire. Instead, depletion was associated with a higher likelihood that an attempt to resist desire would be successful, suggesting that depletion primarily affects the ability to enact behaviour consistent with motivations to control behaviour, rather than increasing the strength of motivation itself (Hofmann et al., 2012). While an "attempt to resist a desire" is not the same as a measure of the strength of motivation to control behaviour, it is likely to be indicative of a certain level of motivation to control behaviour in line with a goal that conflicts with that desire. If ego depletion indeed reduces the motivation to control behaviour, as is suggested by the process model, the likelihood of attempting to resist a desire that conflicts with a valued goal would also be reduced by ego depletion. However, this was not the case in the findings reported by Hofmann et al. (2012).

In contrast, one study measured a variable related to the motivation to control behaviour, and produced results consistent with the process model of ego depletion (D. Walsh, 2014a). In this study, participants were randomised to a 
condition in which they were primed with the goal of healthy eating or a control condition prior to completing a depleting or a non-depleting task. Participants reported their commitment to a goal of healthy eating following the depletion manipulation. The authors did not report a main effect of depletion on goal commitment. However, the effect was moderated by goal priming: in the goal priming condition, depleted and non-depleted participants showed no significant difference in goal commitment; however, in the non-goal priming condition, depletion resulted in lower goal commitment compared to the non-depleted condition. Moreover, lowered commitment to the goal of healthy eating mediated the effect of depletion on consumption of an unhealthy snack food, thus suggesting that the depletion effect is due to a shift in motivation, in support of the process model.

\section{The Current Study}

To date, no study has tested whether ego depletion affects motivation to control behaviour in a specific self-control situation. D. Walsh (2014a) assessed general commitment to a self-control goal, by asking participants to rate the importance of the goal of healthy eating, and how committed and determined they were to achieve that goal. By contrast, evidence for a difference in motivation to control behaviour in a specific situation (e.g. by asking participants to rate their motivation to control or limit their intake of unhealthy food where there was the opportunity to consume that food) would provide stronger support for the process model of ego depletion. According to this theory, depleted individuals would be expected to experience lower motivation to control behaviour specific to a situation following an initial self-control task compared with non-depleted individuals. In contrast to Walsh, Hofmann et al. (2012) tested the interaction between depletion, desire, and self-control attempts, in predicting self-control outcomes, and found support for the reflective-impulsive model across various behavioural domains (e.g. tobacco, spending, media use, eating) with a naturalistic, selfreport study design. The current study, however, sought to test this interaction specifically in a controlled lab-based study of unhealthy food intake. This allowed for control over aspects of the food environment that may influence desire and intake (e.g. food variety, portion size, allowed eating time, etc.) that was not possible in Hofmann et al.'s naturalistic study design.

Specifically, the current study sought to test competing predictions of the reflective-impulsive and process models about the relationships between ego depletion, desire strength, motivation to control behaviour, and self-control. A dual-task paradigm was employed, whereby participants were randomised to complete either an initial depleting or non-depleting task, and then all participants completed a self-control task: a taste-test measure of unhealthy snack consumption. Participants then reported the extent to which they experienced a desire to indulge in the snacks when first presented with them, and the extent to 
which they attempted to control their intake of the snacks during the taste-test. We specifically recruited a sample of young women who held the goal of healthy eating for weight management to ensure that the taste-test would represent a valid test of self-control.

If the reflective-impulsive model (Figure 1) were supported, we would expect that only individuals in a depleted state would show eating behaviour consistent with desires, such that a stronger desire to consume unhealthy food would predict higher snack consumption; while individuals in the non-depletion condition should show eating behaviour consistent with their motivation to control their intake, such that individuals with a higher motivation to control intake would consume less snack food. This would suggest that individuals in the non-depletion condition have the resources to control their intake in line with their motivations and to overcome strong desires to indulge, while the intake of depleted individuals would be more strongly driven by the desire experienced. In contrast, if the process model (Figure 2) were supported, we would expect that depletion would reduce motivation to control snack intake in the taste-test, and that this motivation would mediate the relationship between ego depletion and intake (i.e. that ego depletion would indirectly result in higher intake via reduced motivation). In addition, we would expect that depletion would result in lower selfreported strength of desire to indulge in the foods in the taste-test, and that desire would also mediate the relationship between ego depletion and intake (i.e. that ego depletion would indirectly result in higher intake via increased desire).

\section{METHOD}

\section{Participants}

A sample of 156 women was recruited from the undergraduate student population at Flinders University. An estimate of sample size was determined a priori based on similar studies conducted in our laboratory (Haynes, Kemps, \& Moffitt, 2015a, 2015b). Data collection was conducted over one semester, and

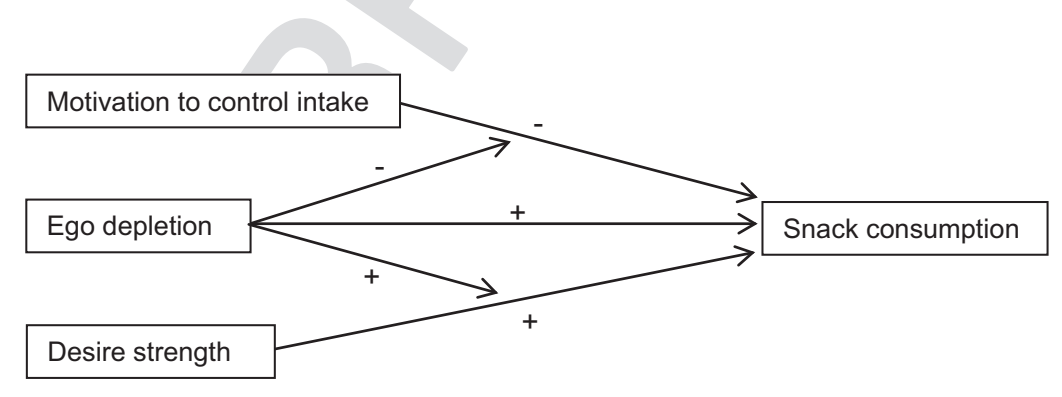

FIGURE 1. Reflective-impulsive model: Ego depletion interacts with desire (impulsive factor) and motivation (reflective factor) to predict subsequent snack consumption. 


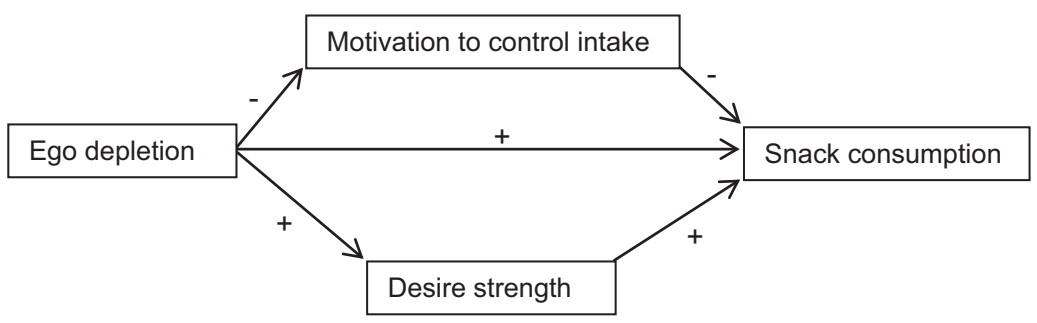

FIGURE 2. Process model: Ego depletion influences snack consumption by increasing desire to indulge, and reducing motivation to control behaviour.

recruitment continued until the end of the semester to offer a buffer for any missing data. Advertisement materials stated that the study aimed to investigate the relationship between attention and taste perception, and asked for female volunteers who were: (a) motivated to eat healthily to manage weight, (b) fluent in English, and (c) had no food allergies or intolerances. The sample was aged between 17 and 25 years $(M=19.53, S D=2.07)$, with a mean body mass index (BMI) of $22.48(S D=3.79)$. The majority of participants were first-year psychology student volunteers who participated for course credit $(n=110)$. The remainder of the sample $(n=46)$ were recruited from the wider undergraduate student population, and received a $\$ 10$ honorarium for participating.

\section{Design}

The experiment employed a between-subject design, with participants randomly assigned to the two depletion conditions (depletion, control). The outcome measures included snack food consumption, and self-reported strength of desire and motivation to control intake.

\section{Materials}

Depletion Task. The e-crossing task was used to manipulate ego depletion (Baumeister et al., 1998; DeWall, Baumeister, Stillman, \& Gailliot, 2007; D. Walsh, 2014b). Participants were told that the task was designed to measure attention to task-relevant stimuli. Participants in both the depletion and nondepletion conditions initially completed the same crossing out task. They were provided with a page of text, and instructed to cross out each instance of the letter "e". In line with previous research, the text was taken from a statistics text, selected for its technical style to minimise interest in the content and distraction from the task (Baumeister et al., 1998). Participants were provided with written instructions, and given the opportunity to ask questions of the experimenter before beginning the task. After 5 minutes, participants were provided with a second set of instructions and a new page of text. Participants in the non-depletion condition were instructed to complete the same crossing out task as they did 
before. Participants in the depletion condition were instructed to cross out each letter "e", except if the "e" was followed immediately by another vowel in the same word, or if a vowel appeared two letters before the "e" in the same word. The complex rule administered in the depletion condition required participants to exercise self-control to inhibit the crossing-out response practised previously. The second task also lasted 5 minutes.

Snack Food Consumption. A taste-test was used to measure consumption of unhealthy snack food. Participants were presented with four bowls of popular energy-dense snack foods (i.e. cheese Twisties, original salted potato chips, M\&Ms, chocolate chip cookies). The placement of the bowls from left to right was counterbalanced across participants using a Latin square procedure with four orders. Participants were instructed to taste some of each of the foods in order to rate their sensory properties (e.g. "How sweet is this product?") on $100 \mathrm{~mm}$ visual analog scales on a rating sheet accompanying each bowl. Participants were informed that they would be given 10 minutes to taste and rate the foods while the experimenter was out of the room, and were advised that they could sample more of the products after the ratings were completed, but were asked not to change their initial ratings. The pre- and post-taste-test weights of the bowls were compared after the participants had left the laboratory in order to gauge intake. Intake in grams was multiplied by the number of kilojoules $(\mathrm{kJ})$ per gram in each food, and summed for the total measure of snack consumption.

Desire Strength. Participants were asked to indicate the extent to which they felt tempted and how much they wanted to eat each of the foods presented during the taste-test on two 7-point Likert scales ranging from 1 (Not at all) to 7 (Completely) for each of the four foods. Mean responses to each question across the foods were calculated, and then the measure of desire strength was calculated as the mean of the two questions, with higher scores indicating a stronger desire to consume the foods.

Motivation to Control Intake during Taste-Test. Participants were asked to indicate the extent to which they were motivated to control or minimise their intake of the snacks presented during the taste-test. Responses were recorded on a 7-point Likert scale, ranging from 1 (Not at all) to 7 (Completely) for each of the four foods. A mean motivation to control intake score was calculated.

Hunger. Self-reported hunger was assessed on a single 7-point Likert scale ranging from 1 (not hungry at all) to 7 (extremely hungry), and was included as a covariate in analyses as it has been shown to affect food consumption (Haynes et al., 2014; Rogers \& Hardman, 2015). 
Depletion Manipulation Check. Participants were asked to indicate the extent to which the second crossing-out-letters task was effortful, exhausting, required concentration, and required them to break a habit. Responses were recorded on four 7-point Likert scales ranging from 1 (Not at all) to 7 (Completely).

\section{Procedure}

Participants completed the experiment individually in a quiet room in the Applied Cognitive Psychology Laboratory. Participants were asked to eat something 2 hours before their scheduled session, and to refrain from eating or drinking anything apart from water until the experiment to equalise hunger levels. After obtaining informed consent, the hunger measure was administered, followed by the e-crossing task and then the taste-test. Finally, the remaining selfreport measures (desire, motivation, age, height and weight) were administered. Measures of desire strength and motivation were administered after the taste-test to avoid contamination of the measure of snack consumption. Data from all participants were included in all analyses.

\section{RESULTS}

\section{Preliminary Analyses and Main Effects of Depletion}

Table 1 presents descriptive statistics for the manipulation check variables, hunger, BMI, and the main outcome measures between the depletion and non-depletion conditions. Independent samples $t$-tests revealed that participants in the depletion condition rated the second e-crossing task as more effortful and exhausting, requiring more concentration, and required them to break a habit to a greater extent than participants in the non-depletion condition. The conditions did not significantly differ on hunger or BMI. Zero-order correlations between all main variables are presented in Table 2 . Hunger was significantly positively correlated with both desire strength and motivation to control intake, and was therefore entered as a covariate in subsequent analyses. ${ }^{1}$ Desire strength was also positively correlated with snack consumption and motivation to control intake. Contrary to predictions, independent sample $t$-tests showed that the conditions

\footnotetext{
1 Hunger has also been shown to correlate with both desire to eat and snack intake in previous research (Haynes et al., 2014; Nederkoorn, Guerrieri, Havermans, Roefs, \& Jansen, 2009). As we were interested in isolating the effects of desire and motivation to control intake, we decided to control for hunger in our main analyses in addition to instructing participants to eat 2 hours before participating to minimise variation in hunger. Excluding hunger as a covariate in analyses did not change the main pattern of results or their significance, except where indicated in footnotes.
} 
TABLE 1

Difference between Depletion and Non-Depletion Conditions on all Variables

\begin{tabular}{|c|c|c|c|c|c|}
\hline \multirow[b]{2}{*}{ Variable } & \multicolumn{2}{|c|}{ Condition } & \multirow[b]{2}{*}{$t^{\mathrm{a}}$} & \multirow[b]{2}{*}{$p$} & \multirow[b]{2}{*}{$d$} \\
\hline & Non-depletion & Depletion & & & \\
\hline Hunger & $3.31(1.60)$ & $3.21(1.40)$ & 0.42 & .67 & 0.07 \\
\hline BMI & $22.37(3.83)$ & $22.58(3.79)$ & 0.34 & .74 & 0.06 \\
\hline \multicolumn{6}{|l|}{ Manipulation checks } \\
\hline Exhausting & $3.19(1.46)$ & $4.01(1.48)$ & 3.48 & .001 & 0.56 \\
\hline Effortful & $4.09(1.68)$ & $4.83(1.45)$ & 2.96 & .004 & 0.47 \\
\hline Requires concentration & $3.63(1.68)$ & $4.36(1.55)$ & 2.82 & $<.01$ & 0.45 \\
\hline Requires breaking a habit & $3.73(1.71)$ & $4.91(1.28)$ & 4.87 & $<.001$ & 0.78 \\
\hline Snack consumption & $887.45(492.30)$ & $847.51(531.94)$ & 0.49 & .63 & 0.08 \\
\hline Desire strength $^{\mathrm{b}}$ & $4.11(1.32)$ & $4.16(1.37)$ & 0.24 & .81 & 0.04 \\
\hline Motivation to control intake ${ }^{c}$ & $3.53(1.49)$ & $3.05(1.35)$ & 2.06 & .04 & 0.34 \\
\hline
\end{tabular}

${ }^{\text {a }}$ degrees of freedom $=154$ for each comparison, except for BMI $(d f=151)$ due to missing data.

${ }^{\mathrm{b}}$ Univariate ANOVA results including hunger as a covariate, $F(2,153)<1, p=.89$, partial $\eta^{2}=00$.

${ }^{\mathrm{c}}$ Univariate ANOVA results including hunger as a covariate, $F(2,153)=4.83, p=.03$, partial $\eta^{2}=03$.

did not significantly differ on desire strength or snack intake (Table 1). However, in line with the process model of ego depletion, participants in the depletion condition reported significantly lower motivation to control intake than those in the non-depletion condition, even when controlling for hunger.

\section{Interactions between Depletion Condition and Both Motivation to Control Intake and Desire Strength in Predicting Snack Consumption}

A moderation analysis was conducted to explore the two-way interactions between depletion and both motivation to control intake and desire strength in predicting snack consumption, using the SPSS macro PROCESS (Hayes, 2012). ${ }^{2}$ The overall model was significant, $R=.38, F(6,149)=4.19, p<.001$. Both motivation and desire significantly predicted snack consumption, such that participants with higher motivation to control intake ate less snack food, $B=-134.89, t(149)=3.22, p=.002$, and those reporting higher desire consumed more snack food, $B=116.52, t(149)=2.55, p=.01$. Depletion condition did not predict snack foed, $B=-82.14, t(149)=1.04, p=.30$. As predicted, there was a significant interaction between depletion condition and

\footnotetext{
2 Depletion condition was coded as $0=$ control (non-depletion), $1=$ depletion condition, and continuous predictors (hunger, motivation, and desire) were mean centred prior to PROCESS analyses.
} 
TABLE 2

Correlations between Hunger, BMI, Snack Consumption, Desire Strength, and Motivation to Control Intake

\begin{tabular}{llcccc}
\hline \multicolumn{1}{c}{ Variable } & 1 & 2 & 3 & 4 & 5 \\
\hline 1 & Hunger & -.01 & .12 & $.24^{* *}$ & $.20^{*}$ \\
2 & BMI & & .05 & -.08 & .00 \\
3 & Snack consumption & & & $.26^{* *}$ & -.07 \\
4 & Desire strength & & & & $.33^{* * *}$ \\
5 & $\begin{array}{l}\text { Motivation to control } \\
\text { intake }\end{array}$ & & & & \\
\hline
\end{tabular}

${ }^{*} p<.05 ; * *<.01 ; * * * p .001$.

motivation, $B=134.88, \quad t(149)=2.26, \quad p=.03, \quad R^{2}$ change $=.03, \quad F(1$, $149)=5.09 .^{3}$ Simple slopes analysis exploring the relationship between motivation and intake between depletion conditions revealed that in the non-depletion condition, higher motivation predicted lower snack consumption, $B=-136.25$, $t(149)=3.228, p=.002$, while in the depletion condition, motivation did not significantly predict consumption, $B=-0.01, t(1490)=0.001, p=.99$ (Figure 3). However, the interaction between desire and ego depletion was not significant, $B=6.02, t(149)=0.09, p=.92, R^{2}$ change $=.001, F(1,149)=.01$, indicating that the relationship between desire and intake did not vary depending on the level of ego depletion.

\section{Indirect Effect of Ego Depletion on Snack Consumption via Both Motivation to Control Intake and Desire Strength}

To test whether there was an indirect relationship between ego depletion and intake via motivation to control intake and/or desire, PROCESS (Hayes, 2012) was used to conduct a parallel mediation analysis using 5,000 bootstrap samples. The independent variable (depletion condition) and covariate (hunger) were regressed on both mediating variables separately, indicating that depletion was associated with motivation, showing that depletion was associated with lower motivation to control intake, model $R=.27, F(2,153)=5.85, p=.004$, $B=-0.49, t(153)=2.20, p=.03$, but was not associated with desire, model $R=.24, F(2,153)=4.68, p=.01, B=.03, t(153)=0.14, p=.89$. A second regression model was estimated, regressing the independent variable, mediators,

\footnotetext{
3 The interaction between motivation and ego depletion was only marginally significant when hunger was not included as a covariate, $B=114.77, t(150)=1.96, p=.05, R^{2}$ change $=.02, F(1$, $150)=3.86$.
} 


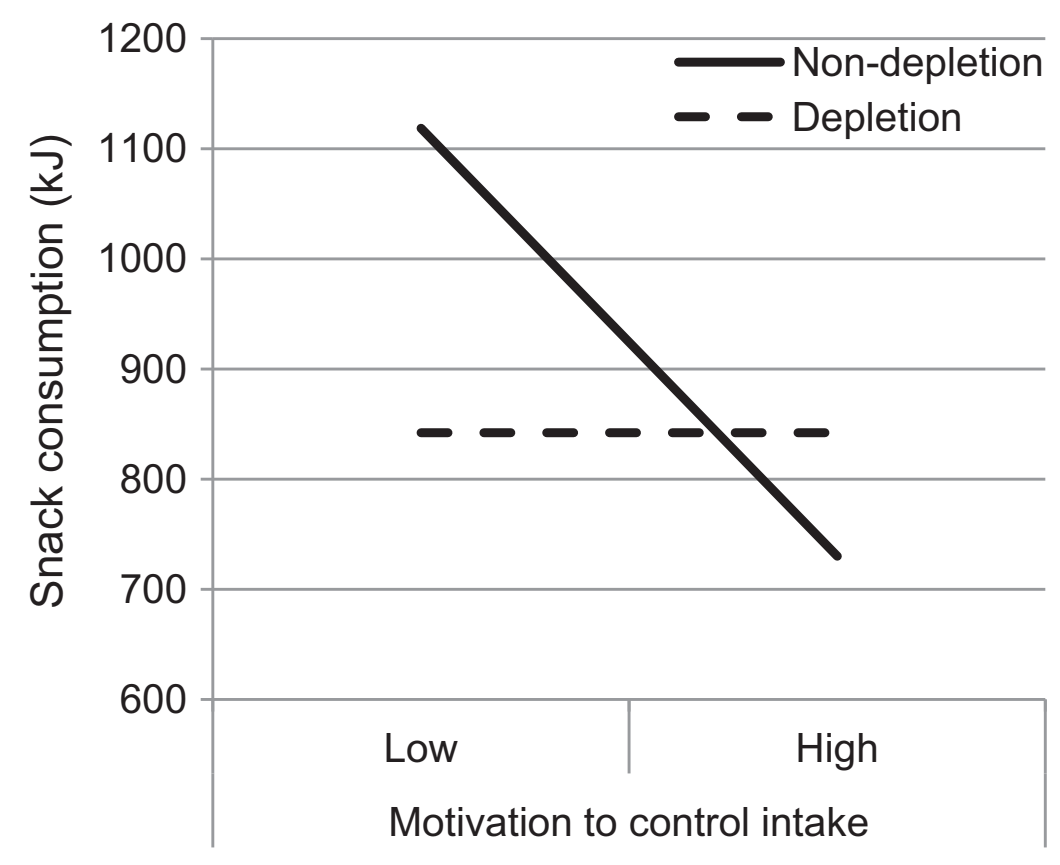

FIGURE 3. Interaction between motivation to control intake and depletion condition in predicting snack consumption.

and covariate on the outcome variable (snack consumption), $R=.33, F(4$, $151)=4.64, p=.002$. Higher snack consumption was predicted by lower motivation to control intake, $B=-71.51, t(151)=2.41, p=.02$, higher desire, $B=118.00, \quad t(151)=3.73, \quad p=.001$, but not by depletion condition, $B=-82.91, t(151)=1.04, p=.30$. The indirect effect of depletion on snack consumption through motivation was $35.18(S E=24.32)$. The $95 \%$ confidence interval around the estimated indirect effect did not contain zero $(3.24,103.40)$, indicating that ego depletion had an indirect effect on higher intake via motivation to control intake. The indirect effect of depletion on snack consumption through desire was $3.45(S E=26.06)$, and the $95 \%$ confidence interval around the indirect effect contained zero $(-52.31,54.22)$, indicating that ego depletion did not have a significant indirect effect on intake via desire strength.

\section{DISCUSSION}

The current study sought to test competing predictions of the reflective-impulsive and process models about the relationships between ego depletion, desire strength, motivation to control behaviour, and self-control in an eating domain. Results provided partial support for both models. Specifically, in support of the process model, depletion indirectly resulted in higher snack consumption through the influence of lower motivation to control intake. However, the prediction that depletion would result in stronger desire for the snack foods offered during the taste-test was not supported. The findings were also partially 
consistent with traditional accounts of self-control such as the reflective-impulsive model. Specifically, only for participants in the non-depletion condition did higher motivation to control intake correlate with lower snack consumption (although the interaction between motivation and ego depletion was only marginally significant when hunger was not controlled for). In line with the reflective-impulsive model, it was also predicted that desire would predict intake only when participants were depleted; however, on the contrary, desire strength predicted higher snack intake across both conditions.

\section{Ego Depletion Effects on Desire and Motivation to Control Behaviour}

Consistent with the process model of ego depletion, depleted individuals reported lower motivation to control intake than those in the non-depletion condition, when controlling for hunger. This reduced motivation also mediated the relationship between depletion and higher snack intake. While Hofmann et al. (2012) found no significant difference in the likelihood of a self-control attempt between depleted and non-depleted participants, Walsh (2014a) did find that depletion decreased participants' ratings of commitment, determination, and importance of the goal of healthy eating. The current findings are consistent with those of Walsh, and extend them in an important way. In contrast to Walsh, we specifically sought to recruit individuals who were motivated to manage their weight through healthy eating to ensure that the taste-test presented a self-control dilemma. Since depletion has a temporary effect on self-control performance, we thought it important to investigate situation-specific motivation by asking participants to rate their motivation to control or limit their intake of unhealthy food during the taste-test. In contrast, Walsh assessed general motivation toward the goal of healthy eating. If the process model is accurate, we would expect a difference in motivation to control behaviour specific to the self-control test presented, which was supported in the current study. Further research which simultaneously examines both general goal commitment and situation-specific motivation would be valuable to test how they interact, and to determine whether they are differentially impacted by ego depletion.

The finding that ego depletion did not increase the strength of desire to consume the unhealthy foods offered during the taste-test is inconsistent with the process model. Some studies have shown that depletion increases incentive sensitivity, and increases neural activity in reward-related centres among dieters when exposed to food cues (Schmeichel et al., 2010; Wagner et al., 2013). However, other studies have revealed differences in the strength of the relationship between hedonic processing of food cues and self-control of eating behaviour between depleted and non-depleted individuals (e.g. Friese et al., 2008; Hofmann et al., 2007). The current study was the first to assess self-reported desire between depleted and non-depleted participants. Taken together with previous 
findings, the current results suggest that increased incentive sensitivity and food reward activity in depleted compared to non-depleted individuals may not necessarily translate into increased subjective desire to indulge in unhealthy but immediately gratifying snack foods.

\section{Interactions: Ego Depletion, Desire, and Motivation to Control Behaviour}

Consistent with the reflective-impulsive model, the current study showed that ego depletion interacted with motivation to control intake to predict actual intake during the taste-test (i.e. self-control outcome). Only in the depletion condition did a higher motivation to control intake predict lower snack consumption. These results suggest that when not depleted, individuals have the resources to enact behaviour consistent with the reflective system. However, regarding the moderation of the impulsive system on behaviour (i.e. influence of desire), the reflective-impulsive model was not supported: desire significantly predicted intake regardless of condition assignment. The current pattern of results regarding the interaction between ego depletion and motivation to control behaviour (but not desire strength) mirrored that of Hofmann et al.'s (2012) experience sampling study. Specifically, they found that participants who had not recently enacted self-control (i.e. who were not depleted) were better able to enact behaviour consistent with their attempts to self-control, and to overcome desire. In contrast, when depleted, participants' attempt to control behaviour was not predictive of their behaviour, which was instead more strongly predicted by their immediate desires. The current study adds to this evidence for the interaction between ego depletion and motivation in the eating domain, by testing these interactions using a lab-based study design which allowed experimental control over aspects of the food environment.

\section{Other Findings}

Both self-reported hunger and desire for snack food were found to correlate with a higher motivation to control intake. While we had no specific predictions about this relationship, it is nevertheless an interesting finding. Some previous studies have revealed that exposure to a strong "temptation" cue can enhance self-control by bolstering motivation toward the goal that is threatened by that temptation. For example, Kroese and colleagues found that exposure to strong temptations increased the accessibility of a dieting goal, strengthened goal intentions and self-reported goal importance, and facilitated goal-focused behaviour (Kroese, Evers, \& De Ridder, 2009, 2011). The authors suggested that individuals may underestimate the potential effect of weak temptations on consumption, while in contrast, strong temptations present a clear threat to a valued goal and therefore activate stronger intentions and motivations to control behaviour. 
Similarly, the experience of stronger desire in the current study may have facilitated the recognition of the taste-test as a threat to the goal of healthy eating for weight management, thus explaining the correlation between desire and higher motivation to control intake. However, it is difficult to draw conclusions about causal directions as both desire and motivation were measured using retrospective self-report measures. Disentangling the relationship between desire and motivation, and investigating why and under which conditions stronger desire or temptation may benefit motivation toward a conflicting goal, would be a useful direction for future research.

\section{Questioning the Ego Depletion Effect}

The current study did not find a main effect of ego depletion on snack consumption. In addition, when individuals were depleted, snack consumption remained relatively low under both high and low motivation to control behaviour (Figure 3). These observations speak to the current debate about the size and robustness of the ego depletion effect on self-control (Carter \& McCullough, 2014; Hagger et al., 2015). A number of studies have failed to show effects of ego depletion on tasks thought to reflect self-control, such as general working memory performance and financial decision-making (Alós-Ferrer, Hügelschäfer, \& Li, 2015; Lurquin et al., 2016). Others present caveats to the ego depletion effect. For example, Job et al. (2010) demonstrated that only individuals who believe that willpower is a finite resource are susceptible to ego depletion. These, and the current findings, highlight a clear need for further research on the conditions under which ego depletion affects subsequent self-control. In particular, a focus on the combined effects of ego depletion and other moderating factors might provide a more promising avenue for advancing the literature on self-control.

\section{Practical Implications}

In a practical sense, the findings of the current study suggest that reducing desire for indulgence in unhealthy stimuli may be the best way to facilitate healthy eating behaviour, regardless of self-regulatory resources. Only for participants in the non-depletion condition did a high motivation to control intake reduce snack food consumption. However, desire was a significant predictor of snack consumption across conditions, suggesting that desire may affect behaviour even when individuals possess higher levels of self-regulatory resources. Therefore, individuals might be better placed to deal with daily challenges of self-control by managing desire and temptation (e.g. limiting exposure to foods that are routinely craved, distracting oneself from the desire or craving for unhealthy snack foods) rather than focusing on ways of maintaining strong motivation toward one's goals (e.g. reminding oneself of a valued goal). Likewise, an appropriate focus for interventions that are effective at facilitating healthy behaviour across 
situations could be to reduce desire for unhealthy stimuli. For example, previous studies have suggested that changing the way appetitive stimuli are processed at an automatic level could reduce desire for, and subsequent intake of, unhealthy food. In particular, chocolate cravings have been shown to be related to an attentional bias for chocolate-related cues, and retraining attentional biases away from those cues can reduce both cravings and subsequent intake (Kemps \& Tiggemann, 2009; Kemps, Tiggemann, Orr, \& Grear, 2014). Similarly, positive implicit evaluations of unhealthy food have been shown to predict stronger experienced temptation to indulge in unhealthy food and subsequent intake (Haynes et al., 2014). While some studies have shown that retraining those implicit evaluations can produce healthier food choices across all individuals (Hollands, Prestwich, \& Marteau, 2011; E.M. Walsh \& Kiviniemi, 2014), others have shown that retraining implicit food evaluations may only reduce temptation strength and intake of unhealthy food among individuals with low inhibitory control (Haynes et al., 2015a, 2015b). Future research should further explore strategies to reduce the desire for unhealthy but immediately rewarding stimuli, in order to facilitate goal-consistent behaviour.

\section{Limitations and Future Directions}

Several limitations to the current study should be acknowledged. The study implemented a laboratory-based test with a view to control variables not possible in a previous investigation of the interactions between ego depletion, motivation, and desire in an ecological momentary assessment design (Hofmann et al., 2012). However, it remains to be seen whether the effects reported here generalise to the experience of self-control over unhealthy snacking in the "real world". Furthermore, motivation and desire were assessed using retrospective self-report measures. In future, experimental manipulation of ego depletion and/ or motivation and desire might be combined with more ecologically valid approaches to the assessment of self-control of behaviour (e.g. ecological momentary assessment). This would provide insight into whether the same effects found here can be replicated in "real-world" settings, while overcoming some of the limitations of self-report measures of desire and motivation. Finally, future research could extend the current between-subjects design to include a within-subjects comparison of motivation to control behaviour before versus after the ego depletion manipulation. This would enable tracking of within-person shifts in motivational priorities as a result of ego depletion in line with predictions of the process model.

\section{Conclusion}

In conclusion, the current study tested alternative predictions about ego depletion drawn from the process and reflective-impulsive models in an eating 
domain. Findings revealed partial support for the process model: ego depletion reduced the motivation to control intake, which in turn increased snack intake, but ego depletion did not increase desire for appetitive stimuli. We also found partial support for the reflective-impulsive model. Specifically, only when not depleted were individuals able to act according to their motivation to control intake. However, depletion did not significantly moderate the relationship between desire and snack consumption, suggesting that desire may be an appropriate focus for facilitating healthy behaviour across different self-control conditions.

\section{REFERENCES}

Alós-Ferrer, C., Hügelschäfer, S., \& Li, J. (2015). Self-control depletion and decision making. Journal of Neuroscience, Psychology, and Economics, 8 (4), 203-216. doi:10.1037/npe0000047

Baumeister, R.F., Bratslavsky, E., Muraven, M., \& Tice, D.M. (1998). Ego depletion: Is the active self a limited resource? Journal of Personality and Social Psychology, 74 (5), 1252-1265. doi:10.1037/0022-3514.74.5.1252

Carter, E.C., \& McCullough, M.E. (2014). Publication bias and the limited strength model of self-control: Has the evidence for ego depletion been overestimated? Frontiers in Psychology, 5, 823. doi:10.3389/fpsyg.2014.00823

Deutsch, R., \& Strack, F. (2006). Duality models in social psychology: From dual processes to interacting systems. Psychological Inquiry, 17 (3), 166-172. doi:10.1207/ s15327965pli1703_2

DeWall, C.N., Baumeister, R.F., Stillman, T.F., \& Gailliot, M.T. (2007). Violence restrained: Effects of self-regulation and its depletion on aggression. Journal of Experimental Social Psychology, 43 (1), 62-76. doi:10.1016/j.jesp.2005.12.005

Dube, L. (2007). Implicit and explicit influences on spontaneous and deliberate food choices. Advances in Consumer Research, 34, 104-109. doi:10.1037/14333-005

Friese, M., Hofmann, W., \& Wanke, M. (2008). When impulses take over: Moderated predictive validity of explicit and implicit attitude measures in predicting food choice and consumption behaviour. British Journal of Social Psychology, 47, 397-419. doi:10.1348/014466607X241540

Fujita, K. (2011). On conceptualizing self-control as more than the effortful inhibition of impulses. Personality and Social Psychology Review, 15, 352-366. doi:10.1177/ 1088868311411165

Hagger, M.S., Chatzisarantis, N.L., Alberts, H., Anggono, C.O., Birt, A., Brand, R., et al. (2015). A multi-lab pre-registered replication of the ego-depletion effect. Perspectives on Psychological Science, 11 (4), 546-573. doi:10.1177/1745691616652873

Hayes, A.F. (2012). PROCESS: A versatile computational tool for observed variable mediation, moderation, and conditional process modeling [White paper]. Retrieved from http://www.afhayes.com/public/process2012.pdf

Haynes, A., Kemps, E., \& Moffitt, R. (2015a). Inhibitory self-control moderates the effect of changed implicit food evaluations on snack food consumption. Appetite, 90, 114 122. doi:10.1016/j.appet.2015.02.039 
Haynes, A., Kemps, E., \& Moffitt, R. (2015b). The moderating role of state inhibitory control in the effect of evaluative conditioning on temptation and unhealthy snacking. Physiology \& Behavior, 152, 135-142. doi:hof10.1016/ j.physbeh.2015.09.020

Haynes, A., Kemps, E., Moffitt, R., \& Mohr, P. (2014). Reduce temptation or resist it? Experienced temptation mediates the relationship between implicit evaluations of unhealthy snack foods and subsequent intake. Psychology \& Health, 30, 534-550. doi:10.1080/08870446.2014.984713

Hofmann, W., Friese, M., \& Wiers, R.W. (2008). Impulsive versus reflective influences on health behavior: A theoretical framework and empirical review. Health Psychology Review, 2, 111-137. doi:10.1080/17437190802617668

Hofmann, W., Rauch, W., \& Gawronski, B. (2007). And deplete us not into temptation: Automatic attitudes, dietary restraint, and self-regulatory resources as determinants of eating behavior. Journal of Experimental Social Psychology, 43, 497-504. doi:10.1016/j.jesp.2006.05.004

Hofmann, W., Vohs, K.D., \& Baumeister, R.F. (2012). What people desire, feel conflicted about, and try to resist in everyday life. Psychological Science, 23 (6), 582-588. doi:10.1177/0956797612437426

Hollands, G.J., Prestwich, A., \& Marteau, T.M. (2011). Using aversive images to enhance healthy food choices and implicit attitudes: An experimental test of evaluative conditioning. Health Psychology, 30 (2), 195-203. doi:10.1037/a0022261

Inzlicht, M., \& Schmeichel, B.J. (2012). What is ego depletion? Toward a mechanistic revision of the resource model of self-control. Perspectives on Psychological Science, 7 (5), 450-463. doi:10.1177/1745691612454134

Inzlicht, M., Schmeichel, B.J., \& Macrae, C.N. (2014). Why self-control seems (but may not be) limited. Trends in Cognitive Sciences, 18 (3), 127-133. doi:10.1016/ j.tics.2013.12.009

Job, V., Dweck, C.S., \& Walton, G.M. (2010). Ego depletion-Is it all in your head? Implicit theories about willpower affect self-regulation. Psychological Science, 21, 1686-1693. doi:10.1177/0956797610384745

Kemps, E., \& Tiggemann, M. (2009). Attentional bias for craving-related (chocolate) food cues. Experimental and Clinical Psychopharmacology, 17 (6), 425-433. doi:10.1037/ a0017796

Kemps, E., Tiggemann, M., Orr, J., \& Grear, J. (2014). Attentional retraining can reduce chocolate consumption. Journal of Experimental Psychology: Applied, 20 (1), 94-102. doi:10.1037/xap0000005

Kroese, F.M., Evers, C., \& De Ridder, D.T.D. (2009). How chocolate keeps you slim: The effect of food temptations on weight watching goal importance, intentions, and eating behavior. Appetite, 53, 430-433. doi:10.1016/j.appet.2009.08.002

Kroese, F.M., Evers, C., \& de Ridder, D.T.D. (2011). Tricky treats: Paradoxical effects of temptation strength on self-regulation processes. European Journal of Social Psychology, 41, 281-288, doi:10.1002/ejsp.771

Lurquin, J.H., Michaelson, L.E., Barker, J.E., Gustavson, D.E., von Bastian, C.C., Carruth, N.P., et al. (2016). No evidence of the ego-depletion effect across task characteristics and individual differences: A pre-registered study. PLOS ONE, 11 (2), e0147770. doi:10.1371/journal.pone.0147770 
Milyavskaya, M., Inzlicht, M., Hope, N., \& Koestner, R. (2015). Saying "no" to temptation: Want-to motivation improves self-regulation by reducing temptation rather than by increasing self-control. Journal of Personality and Social Psychology, 109, 677693. doi:10.1037/pspp0000045

Muraven, M., \& Baumeister, R.F. (2000). Self-regulation and depletion of limited resources: Does self-control resemble a muscle? Psychological Bulletin, 126 (2), 247 254. doi:10.1037/0033-2909.126.2.247

Nederkoorn, C., Guerrieri, R., Havermans, R.C., Roefs, A., \& Jansen, A. (2009). The interactive effect of hunger and impulsivity on food intake and purchase in a virtual supermarket. International Journal of Obesity, 33 (8), 905-912. doi:10.1038/ ijo. 2009.98

Perugini, M. (2005). Predictive models of implicit and explicit attitudes. British Journal of Social Psychology, 44 (1), 29-45. doi:10.1348/014466604x23491

Rogers, P.J., \& Hardman, C.A. (2015). Food reward. What it is and how to measure it. Appetite, 90, 1-15. doi:10.1016/j.appet.2015.02.032

Schmeichel, B.J., Harmon-Jones, C., \& Harmon-Jones, E. (2010). Exercising self-control increases approach motivation. Journal of Personality and Social Psychology, 99 (1), 162-173. doi:10.1016/b978-0-12-801850-7.00006-8

Strack, F., \& Deutsch, R. (2004). Reflective and impulsive determinants of social behavior. Personality and Social Psychology Review, 8 (3), 220-247. doi:10.1207/ s15327957pspr0803_1

Wagner, D.D., Altman, M., Boswell, R.G., Kelley, W.M., \& Heatherton, T.F. (2013). Self-regulatory depletion enhances neural responses to rewards and impairs top-down control. Psychological Science, 24 (11), 2262-2271. doi:10.1177/0956797613492985

Walsh, D. (2014a). Attenuating depletion using goal priming. Journal of Consumer Psychology, 24 (4), 497-505. doi:10.1016/j.jcps.2014.05.001

Walsh, D. (2014b). Can priming a healthy eating goal cause depleted consumers to prefer healthier snacks? Journal of Consumer Marketing, 31 (2), 126-132. doi:10.1108/ JCM-09-2013-0697

Walsh, E.M., \& Kiviniemi, M.T. (2014). Changing how I feel about the food: Experimentally manipulated affective associations with fruits change fruit choice behaviors. Journal of Behavioral Medicine, 37 (2), 322-331. doi:10.1007/s10865-012-9490-5 